NASA/TM-2012-217199

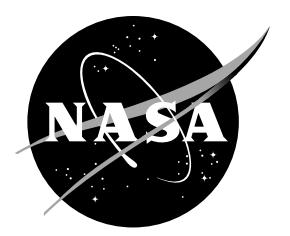

\title{
Tactile Data Entry for Extravehicular Activity
}

Richard J. Adams and Aaron B. Olowin

Barron Associates Inc., Charlottesville, Virginia

Blake Hannaford

University of Washington, Seattle, Washington

O. Scott Sands

Glenn Research Center, Cleveland, Ohio 


\section{NASA STI Program . . . in Profile}

Since its founding, NASA has been dedicated to the advancement of aeronautics and space science. The NASA Scientific and Technical Information (STI) program plays a key part in helping NASA maintain this important role.

The NASA STI Program operates under the auspices of the Agency Chief Information Officer. It collects, organizes, provides for archiving, and disseminates NASA's STI. The NASA STI program provides access to the NASA Aeronautics and Space Database and its public interface, the NASA Technical Reports Server, thus providing one of the largest collections of aeronautical and space science STI in the world. Results are published in both non-NASA channels and by NASA in the NASA STI Report Series, which includes the following report types:

- TECHNICAL PUBLICATION. Reports of completed research or a major significant phase of research that present the results of NASA programs and include extensive data or theoretical analysis. Includes compilations of significant scientific and technical data and information deemed to be of continuing reference value. NASA counterpart of peer-reviewed formal professional papers but has less stringent limitations on manuscript length and extent of graphic presentations.

- TECHNICAL MEMORANDUM. Scientific and technical findings that are preliminary or of specialized interest, e.g., quick release reports, working papers, and bibliographies that contain minimal annotation. Does not contain extensive analysis.

- CONTRACTOR REPORT. Scientific and technical findings by NASA-sponsored contractors and grantees.
- CONFERENCE PUBLICATION. Collected papers from scientific and technical conferences, symposia, seminars, or other meetings sponsored or cosponsored by NASA.

- SPECIAL PUBLICATION. Scientific, technical, or historical information from NASA programs, projects, and missions, often concerned with subjects having substantial public interest.

- TECHNICAL TRANSLATION. Englishlanguage translations of foreign scientific and technical material pertinent to NASA's mission.

Specialized services also include creating custom thesauri, building customized databases, organizing and publishing research results.

For more information about the NASA STI program, see the following:

- Access the NASA STI program home page at http://www.sti.nasa.gov

- E-mail your question via the Internet to help@ sti.nasa.gov

- Fax your question to the NASA STI Help Desk at $443-757-5803$

- Telephone the NASA STI Help Desk at 443-757-5802

- Write to: NASA Center for AeroSpace Information (CASI) 7115 Standard Drive Hanover, MD 21076-1320 
NASA/TM-2012-217199

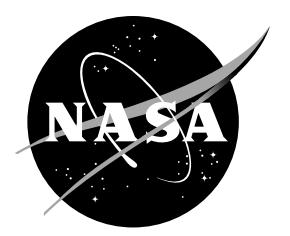

\section{Tactile Data Entry for Extravehicular Activity}

Richard J. Adams and Aaron B. Olowin

Barron Associates Inc., Charlottesville, Virginia

Blake Hannaford

University of Washington, Seattle, Washington

O. Scott Sands

Glenn Research Center, Cleveland, Ohio

Prepared for the

World Haptics Conference 2011

sponsored by the Institute of Electrical and Electronics Engineers

Istanbul, Turkey, June 21-24, 2011

National Aeronautics and

Space Administration

Glenn Research Center

Cleveland, Ohio 44135 


\section{Acknowledgments}

We gratefully acknowledge the support of NASA Glenn Research Center. Work on the Tactile Data Entry System was performed under contract NNX10CC92P1.

Trade names and trademarks are used in this report for identification only. Their usage does not constitute an official endorsement, either expressed or implied, by the National Aeronautics and Space Administration.

Level of Review: This material has been technically reviewed by technical management.

Available from

NASA Center for Aerospace Information 7115 Standard Drive

Hanover, MD 21076-1320
National Technical Information Service 5301 Shawnee Road Alexandria, VA 22312

Available electronically at http://www.sti.nasa.gov 


\title{
Tactile Data Entry for Extravehicular Activity
}

\author{
Richard J. Adams and Aaron B. Olowin \\ Barron Associates Inc. \\ Charlottesville, Virginia 22901 \\ Blake Hannaford \\ University of Washington \\ Seattle, Washington 98195 \\ O. Scott Sands \\ National Aeronautics and Space Administration \\ Glenn Research Center \\ Cleveland, Ohio 44135
}

\begin{abstract}
In the task-saturated environment of extravehicular activity (EVA), an astronaut's ability to leverage suit-integrated information systems is limited by a lack of options for data entry. In particular, bulky gloves inhibit the ability to interact with standard computing interfaces such as a mouse or keyboard. This paper presents the results of a preliminary investigation into a system that permits the space suit gloves themselves to be used as data entry devices. Hand motion tracking is combined with simple finger gesture recognition to enable use of a virtual keyboard, while tactile feedback provides touch-based context to the graphical user interface (GUI) and positive confirmation of keystroke events. In human subject trials, conducted with twenty participants using a prototype system, participants entered text significantly faster with tactile feedback than without $(p=0.02)$. The results support incorporation of vibrotactile information in a future system that will enable full touch typing and general mouse interactions using instrumented EVA gloves.
\end{abstract}

\subsection{Introduction}

The constraints of an EVA suit make conventional modes of human-computer interaction difficult. The environment is inherently mobile, so desktop standards such as a keyboard, mouse, and large liquid crystal displays (LCDs) are unavailable. While adaptations of smaller LCDs and keyboards used in state-of-the-art smartphones are possible, they have not proven to be practical. A small forearmmounted LCD touch screen was previously evaluated in the Electronic Cuff Checklist and flown on several space shuttle missions (Ref. 1). Unfortunately, challenges of glare, small display size, user fatigue, and mobility constraints limited its effectiveness. Restrictions imposed by the space suit glove make interacting with a small keyboard, either real or virtual (touch screen), impractical. These considerations motivate continuing research into modes of human-computer interaction that are better adapted to the constraints and opportunities created by the EVA suit.
To provide crewmembers the same advantages enjoyed by an average smartphone user, future EVA systems may leverage visual, aural, and tactile modes of information exchange. Humans are visually dominant animals, with the ability to process information through the optical channel at an extremely high rate. Graphical display is thus an essential element in an effective human-computer interaction system. The most likely candidate for future EVA systems is the helmet mounted display, studied previously by NASA in a number of settings including simulated Mars environments (Ref. 2). Related work continues today at multiple NASA centers. Closing the loop on this visual element requires the ability to point, select, and move objects. Humans are already "wired" to perform these operations using their hands, making gloves an intuitive bridge into the virtual environment of a GUI.

The gloves required for protection from the harsh EVA environment pose a significant obstacle to efficient data entry for a suited crewmember. Astronauts have consistently indicated a need for better dexterity and feedback (Ref. 3). While NASA has significantly improved glove form, fit, and function from the original 1000 series to the more recent Phase VI design, all EVA gloves share certain common elements. These include an inner bladder to maintain a pressurized environment, a restraint layer to mitigate mechanical loads, and finally a thermal micrometeoroid garment to insulate the crewmember from extreme thermal variations and buffer against the impact of high-velocity particles (Ref. 4). While NASA is striving to make fundamental improvements to future EVA systems, these enduring requirements will almost certainly drive solutions that challenge conventional modes of humanmachine interaction-most notably by stifling the essential element of touch.

Aural presentation, combined with speech recognition, provides bilateral information exchange with the inherent advantage of heads-up and hands-free operation. NASA has tested a spoken dialogue system for human interaction with an autonomous agent, making significant strides to overcoming the challenges of robustness to noise and context discernment (Ref. 5). While these systems promise to be effective in well structured tasks, voice/aural computer interaction is ill-suited 
for communication of spatial information. This limitation makes cumbersome such common tasks as placement of a cursor in a text field or selecting a GUI icon, perhaps explaining why voice recognition systems have made little headway in supplanting the keyboard and mouse (despite being widely available for years on the commercial market). Voice-enabled systems likely have their place in future EVA suits, but will limit human-computer interaction if other data entry modalities are not available.

The sense of touch provides a critical link between humans and our environment. Introducing tactility into a data entry system promises to increase crewmember performance and reduce mental workload. Information displays can overly rely on other channels of perception (especially visual), taxing cognitive resources already heavily burdened by the operational environment. The resulting information overload can result in poor multi-tasking efficiency, poor task performance, and increased frequency of errors (Ref. 6). Touch is a relatively unused sensory pathway in EVA, creating an opportunity to present information without cognitive interference. A tactile glove enables the kind of heads-up operation touch typists use to efficiently key text. In a GUI environment, virtual features can reintroduce physical sensations, such as the feel of pressing a button, to increase throughput and user satisfaction.

A tactile display can recover the sense of touch normally lost behind the layers of a crewmember's gloves. Simple vibrations can provide the illusion of physical contact, rendering phenomena such as texture, impact, and slip. Recent studies make a compelling case for adding tactile cues to mobile devices. In a 2007 investigation, researchers added vibrotactile cues to an HP iPAQ smartphone touch screen keyboard (Ref. 7). The device displayed simple tactile icons to indicate success when a button was pressed correctly or error in the case of a double tap or slip. Subjects executing a text entry task in both a laboratory environment and on a moving train demonstrated higher speeds and reduced errors when presented with the tactile feedback. The users also indicated the touch system reduced mental demand and annoyance level. In related work, researchers found vibratory cues were useful for tasks such as list selection, scrolling, and navigation on a mobile device (Ref. 8).

Several research projects have previously explored use of vibrotactile actuation in gloves for mediating interaction with virtual or physically removed environments. In (Ref. 9), Murray demonstrated the benefits of voice coil-generated vibrotactile information in gloves for teleoperation. Romano et al. integrated tactile feedback into the interior of a protective glove to display information from an externally-mounted slip sensor (Ref. 10). Vibrational cues enabled users to detect surface interactions normally obscured by the thick glove materials. The CyberTouch glove, commercially available system from Cyber Glove Systems LLC, integrates instrumented data gloves with vibrotactile stimulators on the exterior of each finger (Ref. 11). Applications of the system have ranged from industrial prototyping to telecontrol of the
NASA/DARPA Robonaut dexterous humanoid robot (Ref. 12).

This paper describes preliminary experiments employing glove integrated vibrotactile display, gesture recognition, and motion tracking to permit a space suit glove to be used as a data entry device. A prototype system incorporates a plungertype linear electro-magnetic actuator to provide tactile feedback, a MEMS accelerometer to permit registration of button click events, and simple 2-D IR motion tracking to capture hand movements.

\subsection{Prototype System}

\subsection{Surrogate EVA Glove}

Astronauts often don a light moisture-wicking inner glove before putting on the space suit glove. The inner-most glove layer is a rubber pressure bladder. It is followed by a loadbearing restraint layer that, combined with structural reinforcements, maintains a near-constant inner volume. Following are numerous micrometeoroid and thermal protection layers. The outermost soft goods layer is highly resistant to cuts, tears, and abrasion. High wear external surfaces are often coated with a layer of room temperature vulcanized (RTV) rubber (Ref. 4).

Since integration and testing using an actual space suit glove in a pressurized environment was outside of the scope of this initial effort, a surrogate was developed to:

1) Simulate the resistance to finger movement and added inertia of an EVA glove

2) Replicate the rubber pressure barrier, and inner liner (and their dampening effect on vibrotactile sensation)

3) Approximate the impedance loading of the tactile device in a restraint layer

4) Approximate glove kinematics for finger motion sensing

Left and right handed gloves of varying sizes are required for human subject testing. To create reasonable facsimiles, we used three layers of off-the-shelf materials, shown in Figure 1. The first is a cotton under garment. The second is a 20 mil Nitrile rubber glove. The outer layer is a modified soccer goal keeper glove. Right and left handed versions in medium, large, and extra large sizes were created to accommodate a wide range of subjects.
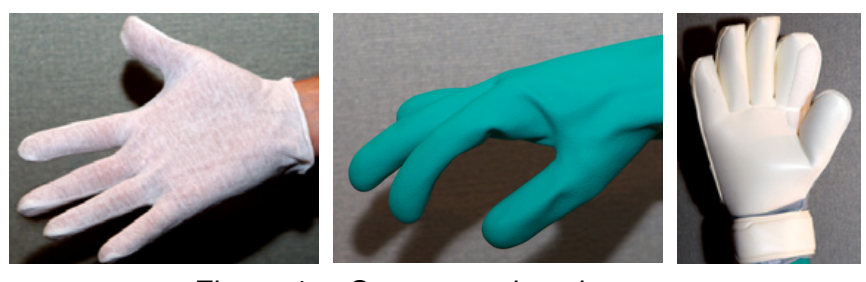

Figure 1.-Surrogate glove layers. 
To permit easy installation of accelerometer and vibrotactile devices between the rubber bladder and outer layer, we modified the outer glove by opening up the top surface from the wrist to the tip of the index finger. Figure 2 shows device placement in the surrogate glove. The upper-right image highlights accelerometer placement above the finger tip and tactile actuator placement below. With the actuator and sensors in place, the glove is reclosed using hook and loop bands (shown lower-right).

\subsection{MEMS Accelerometer "Clicker"}

To enable the system to recognize fingertip "button press" gestures, we combined an Analog Devices ADXL335 MEMS accelerometer with custom embedded software and digital filtering. With the accelerometer attached to the distal phalanx, the user can select on-screen objects by performing a natural tapping motion in free space. An ARM microcontroller samples the analog acceleration output at $100 \mathrm{~Hz}$ and, after processing, generates an HID (human interface device) compliant mouse click event through a USB connection to the host computer.

Figure 2 shows the placement of the accelerometer above the user's index finger. Only a single sensor is used in the prototype, allowing the user to type in a "hunt-and-peck" fashion. The time history in Figure 3 shows accelerometer output during typical use of the system. The green line represents the raw sensor data, while the blue line shows the result of application of a band pass filter to eliminate gravityinduced bias and reduce the impact of high frequency noise. Positive values correspond to the component of fingertip acceleration along a unit vector pointing from the finger pad towards the user's fingernail. When the filtered value crosses the threshold (nominally 0.14 g's), shown as a dotted line, a button click event is generated (highlighted in red).

\subsection{Motion Tracking}

Motion tracking for the prototype system is provided by a CMOS imaging IR sensor (1280 x 480 pixels). The system uses an active array of infrared light emitting diodes to generate backscatter off of a reflective target on the surrogate EVA glove. Tracked glove motions are translated at $100 \mathrm{~Hz}$ into changes in cursor position on the visual display, creating a natural computer mouse-like interface.

The existing sensor is equipped with an optical low pass filter that rejects wavelengths below $800 \mathrm{~nm}$ (and thus the entire visible spectrum). Using its built-in IR LED array for illumination (at $850 \mathrm{~nm}$ ), the system is highly effective at tracking the passive reflective target in normal indoor lighting conditions. In the tracking application, embedded software identifies targets in the streaming IR images of appropriate brightness, size, and aspect ratio. Tracking success is improved by software filtering (to reject pixels below a certain irradiance threshold and discriminate between tracks based on

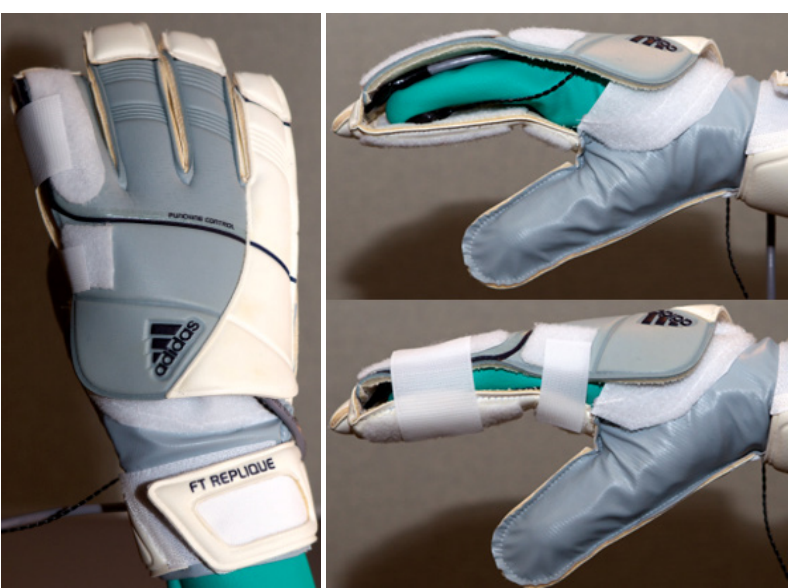

Figure 2.-Device placement in surrogate glove.

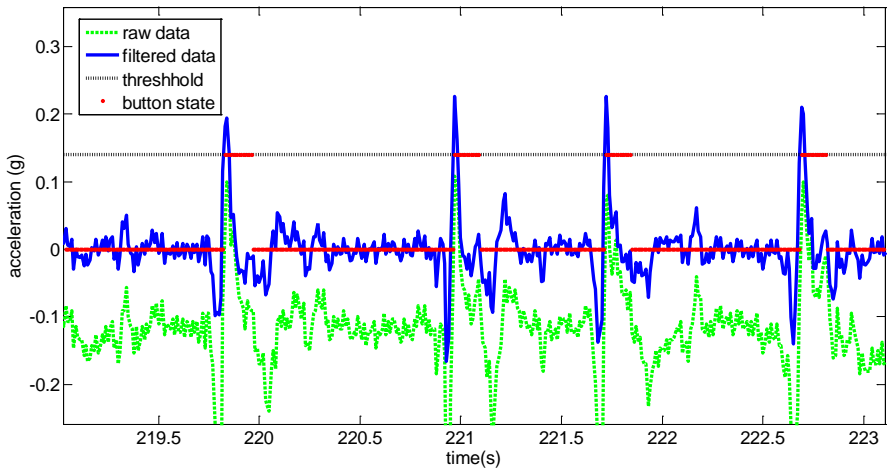

Figure 3.-Accelerometer data in virtual keyboard typing.

target speed) and by optical filtering (to reduce incident radiation outside of the LED illumination band).

It is worth noting that while this approach works well indoors, we are investigating a range of solutions to deal with the added complexities of an outdoor EVA environment. In particular, high solar spectral irradiance in the near IR typically results in saturation of the existing sensor configuration when operated outside on a clear day, even in indirect sunlight (due to local glints and atmospheric scattering). Because this solar "noise" is in-band, additional low pass filtering is ineffective. One potential solution is to increase signal-to-noise by replacing the reflective target on the glove with an active LED. An $850 \mathrm{~nm}$ IR LED integrated in the back of the glove can provide sufficient in-band illumination to "burn through" the ambient IR noise. Combined with additional in-band optical filtering, we have shown this approach to be effective in enabling tracking in outdoor environments under full sun.

A second solution, yet to be explored, takes advantage of the IR sensor's fast frame rate (up to $120 \mathrm{~Hz}$ ) to perform coherent background noise subtraction. By synchronizing the IR camera with a strobed LED, every other frame could show the active target while the odd frames would show only ambient. Pixel-wise subtraction of the two should eliminate 
the vast majority of ambient noise, enabling lower power illumination to achieve the same or better signal-to-noise.

\section{$2.4 \quad$ Vibrotactile Transducer}

Figure 2 shows the placement of the tactile actuator below the pad of user's index finger. The prototype system employs a vibrotactile transducer developed by Engineering Acoustics Inc. (EAI) of Orlando, Florida. The actuator is a plunger-type linear electro-magnetic motor with a small moving contactor that oscillates (at frequencies between 30 to $350 \mathrm{~Hz}$ ) with respect to its outer housing. These plunger-type actuators feature a very fast rise time (time reach 90 percent of peak force output) of $\sim 5 \mathrm{~ms}$ (Ref. 13).The device is tuned to a resonance of approximately $250 \mathrm{~Hz}$, coinciding approximately with the frequency at which the Pacinian corpuscle mechanoreceptors in human skin have maximum sensitivity. A microcontroller board accepts RS-232 commands generated by the virtual keyboard application software running on the host computer, independently controlling pulse frequency, amplitude, and duration.

While a wide range of tactile cues are possible with the EAI tactor, only those representing the most salient features of the keyboard, button presses and key edge crossings, were generated for the test application. The button press cue consisted of two 25 ms pulses with a 25 ms inter-pulse delay. A key edge crossing triggered two 10 ms pulses with a short $5 \mathrm{~ms}$ delay between. Both are rendered using a $250 \mathrm{~Hz}$ modulation frequency. The amplitude of the button press cue is $24 \mathrm{~dB}$ greater than the key edge, creating an approximately fourfold increase in perceived tactile "loudness." Additional effects (such as variable surface roughness) were investigated, but not included due to the potential for generating perceptual confusion.

\subsection{Human Subject Evaluations}

Human subject evaluations were conducted from June 2 through June 11, 2010, at Barron Associates' offices in Charlottesville, Virginia. In the study, participants wearing a surrogate EVA glove were given lines of text and asked to enter them as quickly and accurately as possible using the prototype system. A small LCD monitor rendered a virtual keyboard that the subject used to enter characters through point-and-click operations. Performance and workload were evaluated both with and without tactile feedback.

\subsection{Participants}

Twenty volunteers (14 male and 6 female) participated in the experiment. The subjects were over the age of 21 and without physical impairment that would prevent normal use of their dominant hand. The research program was reviewed and approved by the NASA Committee for the Protection of Human Subjects (CPHS).

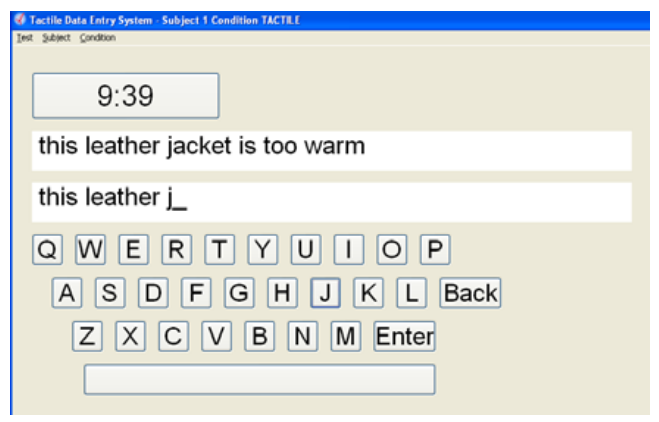

Figure 4.-Virtual Keyboard Application.

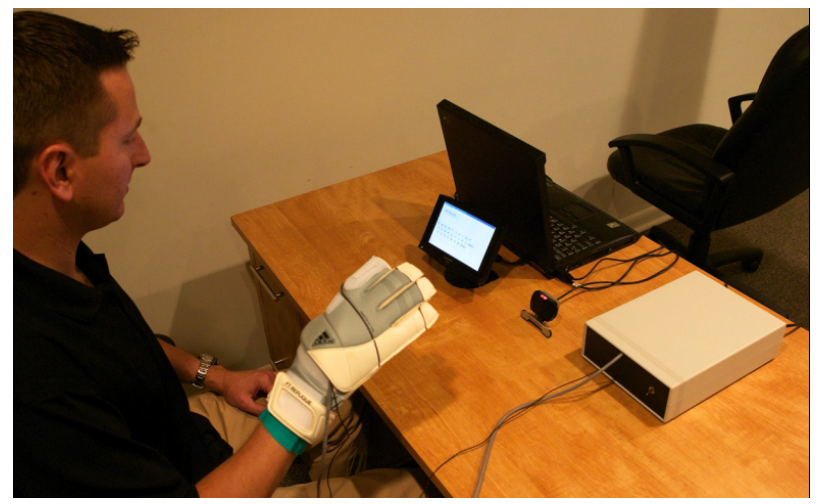

Figure 5.-Integrated system and test setup.

\subsection{Experimental Design and Procedure}

To support human subject evaluations of the prototype system, we developed a Java-based virtual keyboard application that automates human subject data collection and generation of text entry performance measures (Figure 4). During test administration, a pull down menu is used to set the subject number (1-20) and condition ("Practice", "Normal", or "Tactile") before initializing the test. A "START" button then appears and control is passed to the subject. When the subject clicks the start button, it changes into a countdown timer and the first phrase is presented.

The integrated test setup is shown in Figure 5. During test execution, the software draws from a randomized set of 500 phrases, selected to be moderate in length, easy to remember, and containing no punctuation or capital letters (Ref. 14).

When time expires for the given test condition, the code automatically saves four time-tagged files recording the subject's raw keyboard entries (including backspaces represented as an asterisk), the subject's corrected entries, the "target" phrases presented, and the results of text entry performance analysis. Example output of the performance analysis file is shown below.

Raw User $=$ an airpozz ${ }^{* *}$ rt is a very busy place Corrected User $=$ an airport is a very busy place Phrase $=$ an airport is a very busy place MinStringDistance $=0$ 
Raw User = people blow their horns a lot

Corrected User $=$ people blow their horns a lot

Phrase = people blow their horn a lot

MinStringDistance $=1$

(Remaining phrases deleted for brevity)

MSD Sum $=1$

Total Phase Set Character Count $=472$

MSD Per Character $=0.00211864406779661$

Total Raw Character Count $=517$

Total Corrected Character Count $=473$

Key Strokes Per Char $=1.0930232558139534$

Total Corrected Character Count $=473$

Minutes Elapsed $=10.0$

Characters Per Minute $=47.3$

Application software recorded subject entries to generate the dependent variables CPM (the average number of correct characters entered per minute), KSC (average keystrokes per character), and MSD (average mean string distance between target and entered phrases) using published string processing algorithms (Ref. 15). The study had a within subjects design. Each participant entered text using the proposed data entry system under two different conditions.

\section{Condition 1-Normal}

Subjects used their gloved hand to direct the on-screen cursor with no tactile feedback

\section{Condition 2-Tactile}

Subjects used their gloved hand to direct the on-screen cursor and received tactile feedback for button presses and edge crossings

After providing consent and reviewing an informational handout with the principal investigator, subjects donned the surrogate space suit glove on their dominant hand. Both right and left handed units were available in three different sizes. The principal investigator then secured the tactor and accelerometer in the glove index finger with a Velcro strap.

Upon completing a short familiarization exercise, subjects were asked to enter text for 10 minutes in one of the two conditions. Half of the participants were randomly assigned to perform the task under Condition 1 first. The other half of participants performed the sequence in reverse order. At the end of each 10 minute session, subjects completed the NASA Task Loader Index (TLX) to provide an assessment of user workload (Mental Demand, Physical Demand, Temporal Demand, Performance, Effort, and Frustration) (Ref. 16).

\subsection{Results and Discussion}

A paired samples t-test was used to test the null hypothesis that the two conditions have equal effect on the dependent variables. The results are shown in Figure 6. Subjects entered corrected text (CPM) significantly faster with tactile feedback than without $(p=0.02)$. The results suggest that vibrotactile cues aided subjects in effectively using the virtual keyboard. Neither KPC $(p=0.39)$ nor MSD $(p=0.20)$ differed significantly between the two cases.

Observed improvements in data entry performance with tactile cuing are consistent in magnitude with those recorded by Brewster for touch screen applications (Ref. 7). The effect can largely be attributed to the increased confidence provided by positive confirmation of intended keystrokes as well as

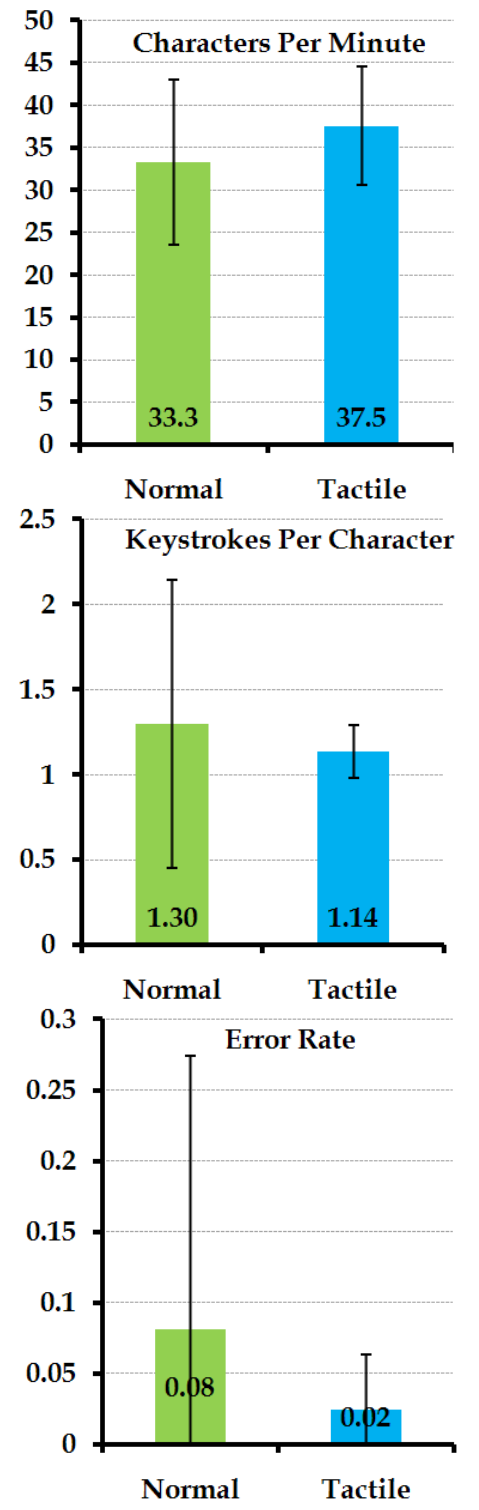

Figure 6.-Results of human subject testing. 
alerting to spurious strikes created by unintentional movements. Note that while average error rate was a factor of four lower in the tactile case, the large between-subjects variance prevents claim of a significant effect. To some extent, this high variability can be attributed to insufficient user training as discussed further below.

Qualitative results from the TLX questionnaire, shown in Figure 7 , are consistent with measured data. Just as participants actually entered more text with tactile cuing, their perceived performance was also significantly greater with tactile feedback than without $(\mathrm{p}=0.01)$. Although the remaining measures all trended in to the advantageous in the tactile case, no claim of significant effect can be made for Mental Demand $(p=0.20)$, Physical Demand $(p=0.44)$, Temporal Demand ( $p=0.37)$, Effort ( $p=0.32)$, or Frustration level $(p=0.16)$. The observed variances in these data point to a need for a larger sample size in future experiments.

The within subjects protocol design (evaluating each subject under both conditions) used in this study was motivated by anticipated high inter-subject variability in performance and expectation of a significant training effect. We randomized presentation of the conditions to control for this effect. Half of the subjects performed Normal first, the other half Tactile. A strong training effect did bear out in test results shown in Figure 8, with subjects on average performing significantly better in their second trial as measured in CPM ( $p<0.01)$.

Ordering also played an interesting role in subjective measures of user workload as captured in the NASA TLX questionnaire (Figure 9). Results demonstrated a significant reduction in Mental Demand $(\mathrm{p}<0.01)$ and Frustration $(p=0.03)$ for the second versus the first trial. It appears as though participants found the gloved data system increasingly accessible as they became more familiar with the interface. Other TLX measures fell below statistical significance, including Physical Demand $(\mathrm{p}=0.80)$, Temporal Demand $(p=0.05)$, Performance $(p=0.13)$, and Effort $(p=0.37)$.

Subjective comments on the questionnaire generally indicated that the addition of tactile feedback created a more natural data entry interface and enhanced ease of use. Some comments however highlighted shoulder or arm fatigue due to the requirement to support the weight of the gloved hand with the forearm in a slightly above-horizontal orientation (see Figure 5). In some cases the fatigue level reduced as the user found a more comfortable position. In others, the cumulative strain made it progressively more difficult to exercise precise control. In hindsight, the physical workload could have been significantly reduced by permitting a more relaxed forearm and shoulder orientation. More importantly, in the targeted EVA application, the motion tracking system will be configured so that crewmembers will be able to maintain a relaxed arm pose while using the gloved data entry interface.

The significance of training effect combined with the reduction of mental workload brought by experience together point to a need for extended familiarization of subjects prior to testing. Providing additional training in human subject evaluations would likely reduce overall variance in subsequent
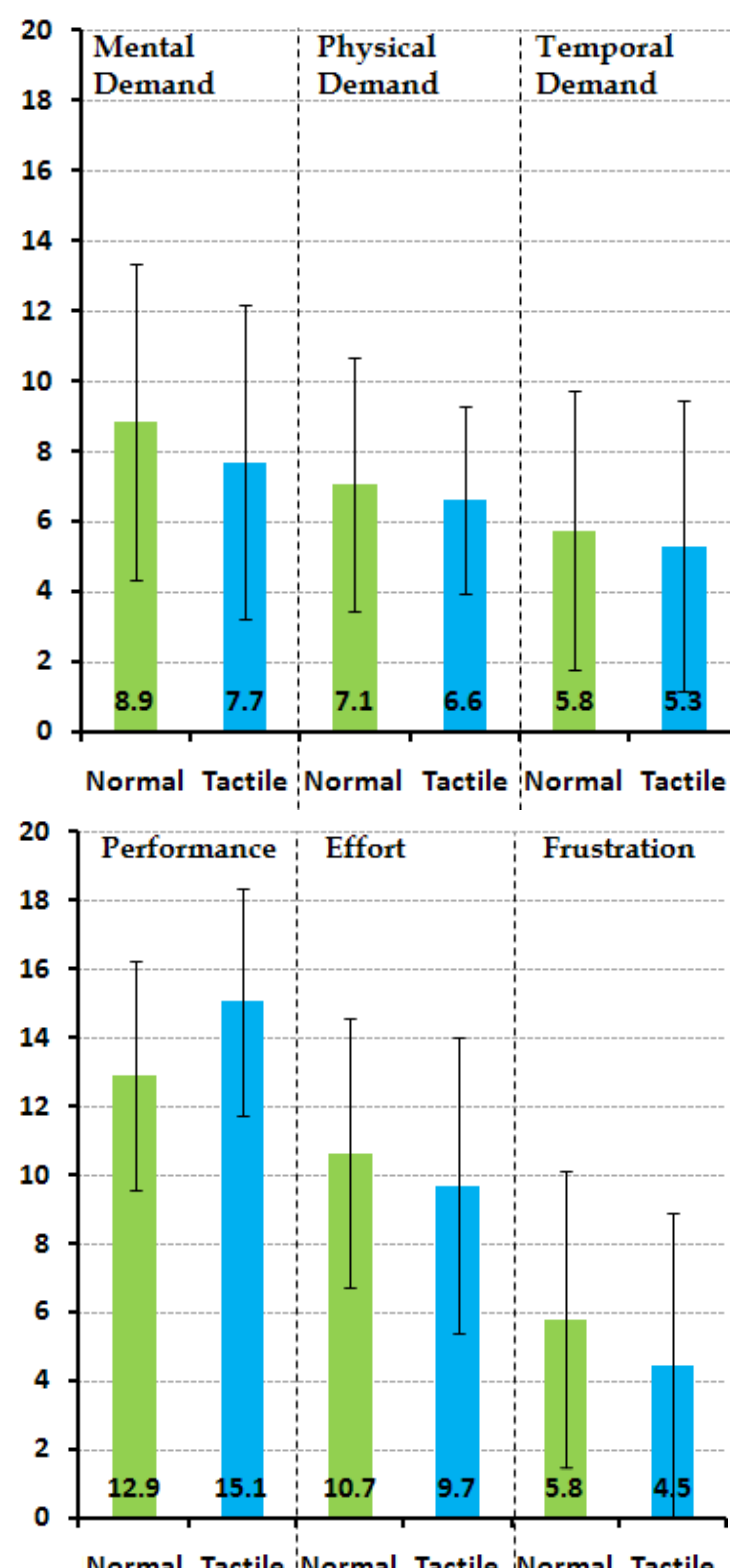

Normal Tactile Normal Tactile Normal Tactile

Figure 7.--Results from NASA TLX questionnaire.

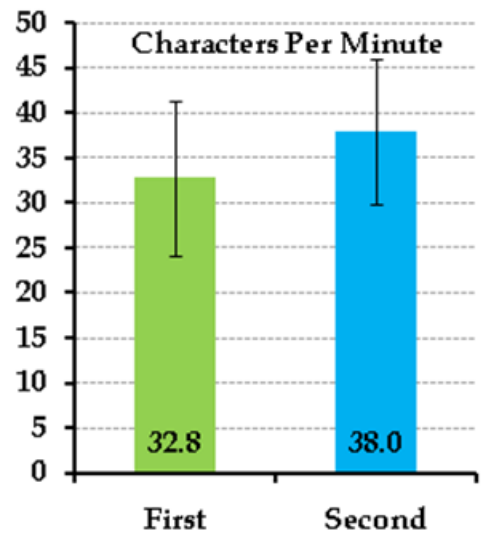

Figure 8.-Impact of training on human subject performance. 


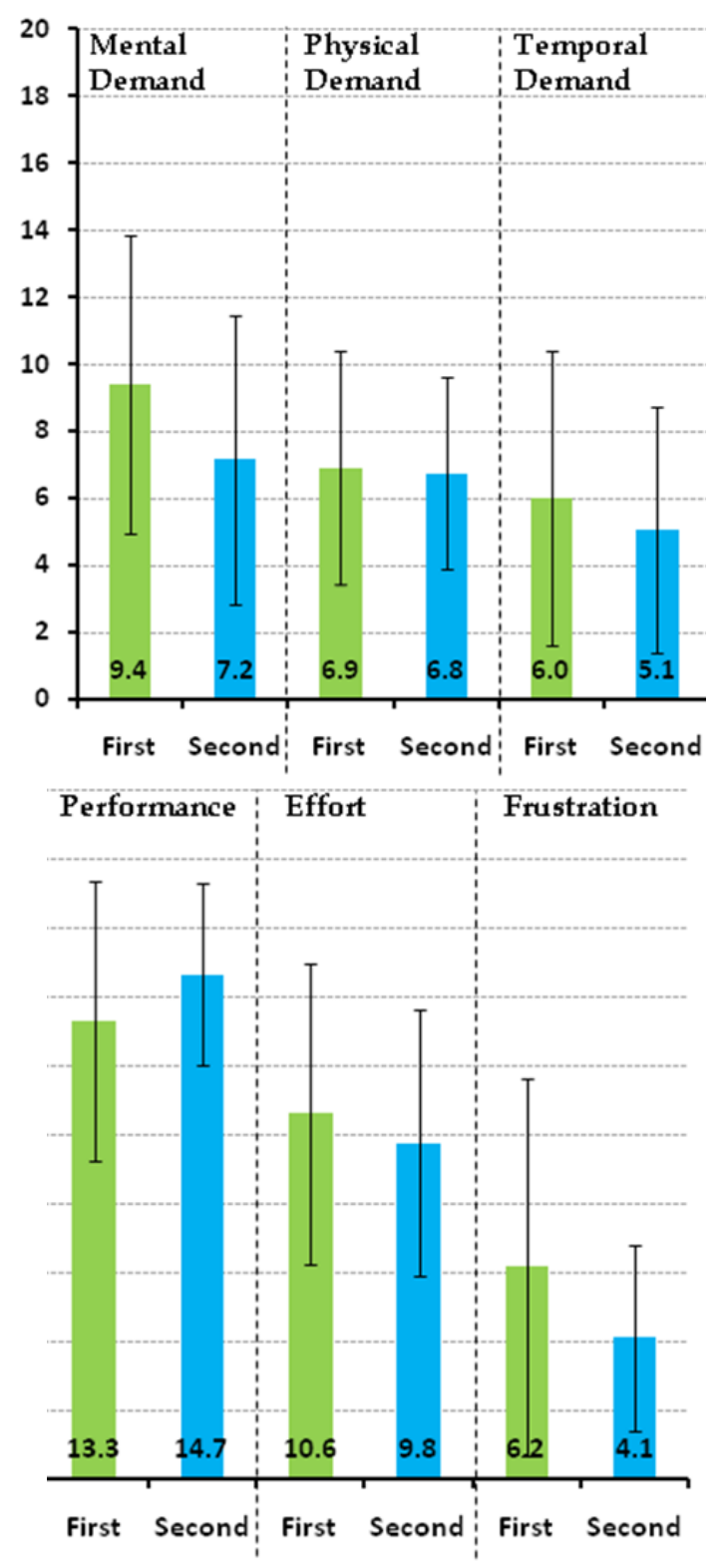

Figure 9.-Impact of training of NASA TLX results.

human subject performance data, increasing the power of the test protocol to distinguish between test conditions. The statistical benefit of this approach will have to be weighed against the increased time required of each subject to realize the training, and the potential for user fatigue.

\subsection{Conclusions and Future Work}

Results of human subject trials using a prototype data entry system provide evidence for the benefit of tactile feedback in enabling a gloved hand to mimic the functionality of a computer mouse as a point-and-click tool. Improvements in text entry performance for a phrase entry task are consistent with those previously observed with introduction of tactile cues to smart phone texting. In future work, we will explore multi-point tracking, multi-finger gesture sensing, and multipoint tactile feedback to enable a more general set of mouse/keyboard interactions. The evolved prototype will integrate the required mechanical elements and electronics into functional EVA gloves to enable testing in a pressurized environment. Follow-on efforts will also include integration of motion tracking in a space suit, addressing sensor placement and ensuring functionality under realistic lighting and line-ofsight limitations. Suited testing will permit evaluation of the tactile data entry hardware in conjunction with helmetmounted display technology currently in development by other researchers under NASA sponsorship.

\section{References}

1. Marmolejo, J.A., "An Electronic Cuff Checklist Information Display for Extravehicular Activity,” 26th International Conference on Environmental Systems, Monterey, CA 1996.

2. Hodgson et al., "Requirements and Potential for Enhanced EVA Information Interfaces," 33rd International Conference on Environmental Systems (ICES), Vancouver, B.C., 2003.

3. Hoffman, S., Advanced EVA Capabilities: A Study for NASA's Revolutionary Aerospace Systems Concept Program, NASA/TP—2004-212068, 2004.

4. Graziosi, D., Stein, J., Ross, A., and Kosmo, J., "Phase VI Advanced EVA Glove Development and Certification for the International Space Station," Proceedings of the International Conference on Environmental Systems, 2001.

5. Hieronymus, J. L., "Clarissa Spoken Dialogue System for Procedure Reading and Navigation," IAC-04-T.3.07, NASA Ames Research Center, USA, 2004.

6. Wickens, C.D., "Multiple Resources and Mental Workload," Human Factors, vol. 50, No. 3, June 2008.

7. Brewster, S., Chohan, F., and Brown L., "Tactile Feedback for Mobile Interactions," Proceedings of the SIGCHI Conference on Human Factors in Computing Systems, 2007.

8. Luk, J., Pasquero, J., Little, S., MacLean, K., Levesque, V., and Hayward, V., "A Role for Haptics in Mobile Interaction: Initial Design Using a Handheld Tactile Display Prototype," Proceedings of the SIGCHI Conference on Human Factors in Computing Systems, 2006.

9. Murray, A.M., "Performance Evaluation of a Wearable Vibrotactile Glove for Telemanipulation," Proceedings of the Human Factors and Ergonomics Society Annual Meeting, 2003, pp. 2102-2102.

10. Romano, J.M., Gray, S.R., Jacobs N.T., Kuchenbecker, K.J., "Toward Tactilely Transparent Gloves: Collocated Slip Sensing and Vibrotactile Actuation," Proceedings of the Symposium on Haptic Interfaces for Virtual 
Environment and Teleoperator Systems, Salt Lake City, UT, USA, March 18-20, 2009, pp. 279-284.

11. CyberTouch, Cyber Gloves Systems LLC, http://www.cyberglovesystems.com, accessed March 6, 2011.

12. Diftler, M.A., et al., "Evolution of the NASA/DARPA Robonaut Control System," Proceedings of the IEEE International Conference on Robotics and Automation, Sep. 2003, pp. 2543-8.

13. Mortimer, B. M., Zets, G. A., and Cholewiak, R. W., "Vibrotactile Transduction and Transducers," The Journal of the Acoustical Society of America, 121, 2970-2977.
14. MacKenzie, I.S. and Soukoreff, R.W., "Phrase Sets for Evaluating Text Entry Techniques,” CHI 2003, Ft. Lauderdale, FL, 2003.

15. Soukoreff, R.W. and MacKenzie, I.S., "Metrics for Text Entry Research: An Evaluation of MSD and KSPC, and a New Unified Error Metric,” CHI 2003, Ft. Lauderdale, FL, 2003.

16. Hart, S.G. and Staveland, L.E. "Development of NASATLX (Task Load Index): Results of Empirical and Theoretical Research,” In P.A. Hancock \& N. Meshkati (Eds.), Human Mental Workload Amsterdam: NorthHolland, 1988, pp. 139-183). 



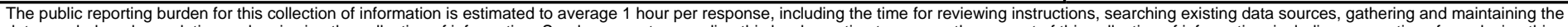

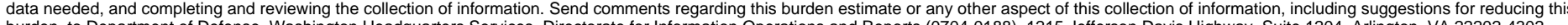

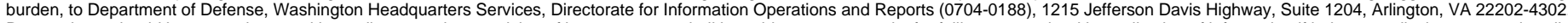

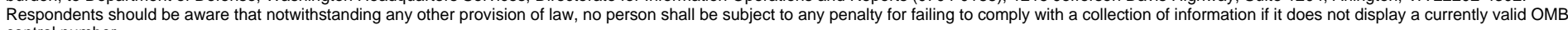

control number.
PLEASE DO NOT RETURN YOUR FORM TO THE ABOVE ADDRESS.

\begin{tabular}{l|l|l}
\hline $\begin{array}{l}\text { 1. REPORT DATE (DD-MM-YYYY) } \\
01-05-2012\end{array}$ & $\begin{array}{l}\text { 2. REPORT TYPE } \\
\text { Technical Memorandum }\end{array}$ & 3. DATES COVERED (FrOm - To)
\end{tabular}

4. TITLE AND SUBTITLE

Tactile Data Entry for Extravehicular Activity

5a. CONTRACT NUMBER

NNX10CC92P1

5b. GRANT NUMBER

5c. PROGRAM ELEMENT NUMBER

6. AUTHOR(S)

Adams, Richard, J.; Olowin, Aaron, B.; Hannaford, Blake; Sands, O., Scott

\section{5d. PROJECT NUMBER}

5e. TASK NUMBER

5f. WORK UNIT NUMBER

WBS 903184.04.20.03.02.01

7. PERFORMING ORGANIZATION NAME(S) AND ADDRESS(ES)

National Aeronautics and Space Administration

John H. Glenn Research Center at Lewis Field

Cleveland, Ohio 44135-3191

\section{SPONSORING/MONITORING AGENCY NAME(S) AND ADDRESS(ES)}

National Aeronautics and Space Administration

Washington, DC 20546-0001
8. PERFORMING ORGANIZATION REPORT NUMBER

E-17884

\section{DISTRIBUTIONIAVAILABILITY STATEMENT}

Unclassified-Unlimited

Subject Category: 54

Available electronically at http://www.sti.nasa.gov

This publication is available from the NASA Center for AeroSpace Information, 443-757-5802

\section{SUPPLEMENTARY NOTES}

\section{ABSTRACT}

In the task-saturated environment of extravehicular activity (EVA), an astronaut's ability to leverage suit-integrated information systems is limited by a lack of options for data entry. In particular, bulky gloves inhibit the ability to interact with standard computing interfaces such as a mouse or keyboard. This paper presents the results of a preliminary investigation into a system that permits the space suit gloves themselves to be used as data entry devices. Hand motion tracking is combined with simple finger gesture recognition to enable use of a virtual keyboard, while tactile feedback provides touch-based context to the graphical user interface (GUI) and positive confirmation of keystroke events. In human subject trials, conducted with twenty participants using a prototype system, participants entered text significantly faster with tactile feedback than without $(\mathrm{p}=0.02)$. The results support incorporation of vibrotactile information in a future system that will enable full touch typing and general mouse interactions using instrumented EVA gloves.

\section{SUBJECT TERMS}

Extravehicular activity; Feedback; Space suits; Gloves

\begin{tabular}{|c|c|c|c|c|c|}
\hline \multicolumn{3}{|c|}{ 16. SECURITY CLASSIFICATION OF: } & \multirow{2}{*}{$\begin{array}{l}\text { 17. LIMITATION OF } \\
\text { ABSTRACT } \\
\text { UU }\end{array}$} & \multirow{2}{*}{$\begin{array}{l}\text { 18. NUMBER } \\
\text { OF } \\
\text { PAGES } \\
16\end{array}$} & \multirow{2}{*}{$\begin{array}{l}\text { 19a. NAME OF RESPONSIBLE PERSON } \\
\text { STI Help Desk (email:help@sti.nasa.gov) } \\
\text { 19b. TELEPHONE NUMBER (include area code) } \\
\text { 443-757-5802 }\end{array}$} \\
\hline $\begin{array}{l}\text { a. REPORT } \\
U\end{array}$ & $\begin{array}{l}\text { b. ABSTRACT } \\
\mathrm{U}\end{array}$ & $\begin{array}{l}\text { c. THIS } \\
\text { PAGE } \\
\text { U }\end{array}$ & & & \\
\hline
\end{tabular}



